\title{
Percutaneous vertebral compression fracture management with polyethylene mesh-contained morcelized allograft bone
}

\author{
Stewart M. Kerr · Benjamin Liechty • \\ Ravi Patel · James S. Harrop
}

Published online: 15 November 2007

(C) Humana Press 2007

\begin{abstract}
Study design A comprehensive systematic review of the literature. Objectives To assess the modern literature on the use of polyethylene mesh-contained morcelized allograft (PMCMA) bone for spinal fusion and vertebral compression fracture management. Summary of background data There are presently no systematic reviews of PMCMA. Methods A systematic literature review was performed within three databases (OVID, PubMed, and Google Scholar) using the following keyword search terms: vertebroplasty, kyphoplasty, vertebral compression fracture, percutaneous, polyethylene mesh, and osteoporosis. Results The initial search identified 764 items, from which two pertinent technique-based articles were identified. There were no published scientific peerreviewed or case series reporting the clinical results of this technique. The use of PMCMA in the management of vertebral compression fractures (VCFs) is similar to vertebroplasty and kyphoplasty. This novel, percutaneous system uses the properties of granular mechanics to establish a conforming, semirigid graft that is purportedly capable of withstanding physiologic loads. Discussion PMCMA is a novel percutaneous technology for the management of VCF and possibly for use as a conforming interbody graft. The available published literature lacks outcome data of the use of PMCMA. Careful, independent research is needed to assess the viability of this technology and its long-term results.
\end{abstract}

S. M. Kerr · B. Liechty · R. Patel · J. S. Harrop $(\bowtie)$

Department of Neurological Surgery, Thomas Jefferson

University Hospital, Philadelphia, PA 19107, USA

e-mail: ejharrop@aol.com
Keywords Vertebral compression fracture . Vertebroplasty · Kyphoplasty · Percutaneous . Polyethylene mesh · Osteoporosis

$\begin{array}{ll}\text { Abbreviations } \\ \text { PMCMA } & \begin{array}{l}\text { Polyethylene mesh-contained morcelized } \\ \text { allograft }\end{array} \\ \text { VCF } & \text { Vertebral compression fracture } \\ \text { PMMA } & \text { Polymethyl methacrylate }\end{array}$

\section{Introduction}

Vertebral compression fractures (VCFs) represent a major cause of morbidity in patients affected by osteoporosis. It is estimated that about 700,000 patients suffer from VCFs each year in the United States and, as the population ages, the incidence is expected to rise accordingly [1,2]. The cost of osteoporotic fractures represents a substantial portion of healthcare spending, with a reported cost of over $\$ 13.8$ billion in 1995 [3]. Out of that total, osteoporotic VCFs are believed to account for $\$ 700$ million per year [1].

Traditional management of symptomatic VCFs has involved a combination of analgesics, bed rest, orthotic bracing, and physical therapy [4-6]. Although these treatments are generally effective, there are indeed cases that require an alternative approach. In recent years, minimally invasive surgical techniques such as vertebroplasty and kyphoplasty have been developed; they are becoming increasingly popular as management options for this common condition. Vertebroplasty is performed in an effort to stabilize the injured bone. It involves the percutaneous injection of bone cement, polymethyl methacrylate (PMMA), into the injured vertebral body. This imparts 
rigidity and stability to the site after the cement has cured, thereby preventing further compression of the affected vertebral body [3, 7]. Kyphoplasty is a more recent percutaneous technique that utilizes a pressurized, inflatable balloon tamp to create a void within the vertebral body. This space is then filled with PMMA $[1,2,8]$. Both techniques appear to be effective, with a high percentage of patients (greater than $90 \%$ ) reporting pain relief. Good functional outcomes are generally observed as well, although kyphoplasty has been more successful in the restoration of vertebral height and kyphotic angle [3-12]. Despite these benefits, both procedures are not without complications.

The Optimesh system was developed by Spineology, Inc. (St. Paul, MN) and recently received FDA-approval as a means for percutaneous vertebral augmentation (PVA). It provides an alternative technique to stabilize and treat VCFs that are refractory to conservative management options.

\section{Methods and results}

Three databases were searched (OVID, PubMed, and Google Scholar), using varying combinations of the following keywords or terms: vertebroplasty, kyphoplasty, spine fracture, percutaneous, compression fracture, allograft, and Optimesh. These searches resulted in 764, 271, $594,253,7$, and 2 items. These items were manually sorted. Only two items directly related to polyethylene meshcontained morcelized allograft (PMCMA) were found. These two items were technique descriptions and lacked clinical outcome data. The authors of both articles recognized that the next step needed to better assess the role of optimesh in the management of osteoporotic VCFs would be a study of clinical outcomes.

\section{Discussion}

The Optimesh system utilizes a unique design. It uses a mesh bag that is made from a woven biocompatible polyester (Dacron). One important mesh characteristic is that it is porous enough to allow fluid transmission and possibly vascular ingrowth. The pore size, however, is small enough to prevent leakage of the granular bone graft material. It is also capable of expanding upon injection to accommodate for any irregularities in the size and shape of the cavity generated during surgery $[2,13]$.

\section{Technique description}

The patient is placed prone and prepped in the usual sterile fashion on a radiolucent table. Digital C-arm fluoroscopy is necessary for proper anatomical localization of the affected VCF and for instrumentation of the vertebral body.

Next, the guide pin is advanced into the vertebral body via a lateral extrapedicular approach. A dilator is placed over the guide pin and advanced into the vertebral body. Care should be made to ensure that the guide pin is not inadvertently advanced as the dilator is delivered into the VCF site.

Next, the access portal is placed over dilator. The dilator-guide pin assembly is then removed. A drill is used to initiate cavity creation. This initial space is subsequently enlarged with shaper-reamers. These expandable reamers are used to create a cavity, which will ultimately contain the morcelized graft filled mesh $[1,2,13]$. The reamers are available in two sizes (5.8 or $10 \mathrm{~mm}$ diameter), and are capable of producing a cavity of various diameters $(14,22$, and $25 \mathrm{~mm}$ diameter) [2].

This is followed by insertion of the woven Optimesh implant sac. The tools and techniques required to implant the graft material have been uniquely developed. A bone fill-tube, which inserts into the mesh bag provides a channel for delivery of graft material. A bone push-rod fits into the fill tube to both deliver and gradually compact the graft material $[1,2]$. In this manner, the sac is densely filled with morcelized bone graft. With the bone graft location confirmed with fluoroscopy, the instruments are removed. The wounds are irrigated and subsequently closed.

\section{Comparison to vertebroplasty and kyphoplasty}

Compression of the paste-like bone graft preparation during delivery causes exudation of the liquid component into the surrounding porous bone. This leaves only the morcelized graft behind in the mesh-containment bag. During this maneuver, the physical properties of the graft preparation change from that of a semisolid paste to a solid that more closely mimic bone [2, 13]. Furthermore, this is achieved without the risk of high-pressure injection that is needed to insert PMMA. Another advantage over PMMA is that the porous graft containment system has the potential to be both osteoconductive and osteoinductive [1, 2 13].

Optimesh is intended to provide a minimally invasive surgical option to treat various forms of intraosseous vertebral insufficiency, most commonly osteoporotic fractures. Although clinical studies remain to be done, there appear to be several perceived advantages to Optimesh over vertebroplasty/kyphoplasty in regards to complication avoidance. Known risks associated with the use of vertebroplasty and kyphoplasty include PMMA emboli, neurological compression from intracanal cement leak, and foreign body reaction $[1,2,4-6,9,10]$. 
Many of the complications reported with vertebroplasty/ kyphoplasty are directly related to the use of PMMA. Leakage of cement into the venous system has been reported and may lead to pulmonary embolism, pulmonary cement embolism, or cerebral vascular cement embolism [1, 2, 4-6]. Neurological damage has also been reported. Acute paraplegia from cement leakage compressing the spinal cord has been reported $[1,2,4,5]$. In addition, the modulus of elasticity of PMMA in the treated level causesa mismatch between it and the adjacent untreated levels. The transition zone between treated and unreated levels causes a so-called stress-riser effect, which has been through to result in an increased incidence of adjacent level compression fractures $[9,10]$. Reports of adjacent fractures for vertebroplasty have been reported to be between $12 \%$ and $52 \%$, and between $3 \%$ and $29 \%$ for kyphoplasty $[1,6,9]$.

Foreign-body reactions to PMMA have also been reported [5]. Another criticism of the use of PMMA in close proximity to the spinal cord is the heat released during the exothermic reaction with cement curing [2, 5]. Although there is experimental evidence in animal models to show the potential for neural tissue damage due to heat, there has not been a reported case of this complication in humans at the time of this writing $[2,5]$. It should be noted that reports of poor outcome for both vertebroplasty and kyphoplasty fluctuate greatly.

The material and biological characteristics of morcelized bone are among the major reasons for the perceived advantages of Optimesh. Compressed allograft bone can be expected to more closely mimic the physical properties of the surrounding native bone $[1,2]$. If the stiffness of vertebral bodies infiltrated with PMMA is in fact the cause of an increased risk of subsequent VCF, the use of bone as graft material may be useful in adjacent vertebrae fracture risk reduction. Due to the porous nature of the woven mesh bag and the morselized bone, it is conceivable that revascularization may occur through and around the graft [2].

\section{Proposed clinical indications}

The criteria for the application of Optimesh for PVA have been described previously [1]. The major indication is VCFs with pain [1, 2]. Although it is not currently approved for interbody use, it has been anecdotally used to effect interbody fusion for the treatment of degenerative disk disease [13]. The complete list of indications and contraindications described by Chiu and Stechison [1] is as follows:

Indications

1. Management of painful osteoporotic or posttraumatic VCFs.
2. Intractable, focal band-like radicular pain that is worse with weight bearing and is relieved with rest or in a recumbent position.

3. Pain refractory to analgesics and narcotics.

4. Osteoporosis, aggressive hemangioma, metastatic disease, osteogenic imperfecta, trauma, or vertebral osteonecrosis resulting in a painful compression fracture.

5. For the management of chronic nonunion following traumatic vertebral body fractures

6. Internal stabilization of unstable posttraumatic VCFs.

7. Management of multiple compression fractures in a patient with poor pulmonary reserve.

Absolute contraindications

1. Patients with painless asymptomatic stable VCFs.

2. Massive "burst" osteoporotic or nonosteoporotic fractures.

3. Patients with fracture(s) that are clearly responding to medical therapy.

4. Osteomyelitis of target vertebra.

5. Prophylactic treatment with no evidence of fracture.

6. Uncorrected coagulation/bleeding disorder.

Relative contraindications

1. Medically high-risk or unstable patient.

2. Patients with retropulsed bone causing spinal canal compromise of greater than $20 \%$.

3. Restless patient (unable to lie prone for the entire procedure under IV conscious sedation).

4. Patient with pain due to herniated spinal disk, facet arthropathy, spinal stenosis, or other degenerative condition.

5. Pathological fracture with tumor significantly extending into the spinal canal [1].

Clinical outcomes

Optimesh has been recently approved for use. So far, no large clinical trials have been reported to date. However, several successful cases have been reported. Chiu and Stechison reported three successful procedures leading to almost immediate postoperative pain reduction [1]. All three patients were of age greater than 70 years, had fractures at different levels, and received conscious sedation and local anesthesia [1]. A CT scan performed seven weeks post-operatively suggested osteointegration at the vertebral body-graft interface in one patient [1].

Modifications to the procedures in kyphoplasty and vertebroplasty may reduce the problems associated with the use of PMMA. The use of calcium phosphate as a graft material has been demonstrated to be osteoconductive [14]. 
However, experimental studies of calcium phosphate grafts have raised concerns of limited ability to convert into bone [14].

Further evidence in material science and animal studies support claims that the compaction of a wet granular material such as morselized bone results in a phase change to a more stable solid form $[13,15]$. The characteristics of this phase change can be likened to the change of properties of dry sand to the properties of wet, packed sand, such as is observed in a sandcastle. This is largely due to increased crowding of particles [15].

There has been little discussion in the literature about the cost effectiveness of surgical management of VCFs, and much of the existing discussion is contradictory. More has been published about the cost effectiveness of vertebroplasty than about other techniques. Although surgery often leads to pain relief where medication has failed, many authors have reached no conclusion about the cost benefit of any procedure. Danish researchers have indicated that the cost of vertebroplasty is offset by the reduction in future hospital visits [16].

Although there is considerable excitement surrounding Optimesh, the data in case reports are not enough to fully assess the outcomes. The elimination of PMMA cement will likely reduce the incidence of several known complications. It is not possible however, to predict if other problems will be generated through the use of Optimesh. Pulmonary thromboemboli and fat emboli have been recorded in vertebroplasty and kyphoplasty, and there is concern that the reaming process and the compacting steps used in Optimesh application may promote the dislodging of bone marrow, which could hypothetically amplify this problem [2]. Additionally, the high degree of fluctuation in the frequency of adjacent compression fractures in both vertebroplasty and kyphoplasty show that the variables leading to this complication, despite many arguments related to vertebral stiffness, have not been completely assessed.

\section{Conclusions}

Optimesh is a new technology. Although a few successful cases have been reported, these do not constitute a statistically significant sample set to make any definitive statements about the safety and general outcomes following PMCMA application. However, given both the initial successes seen in some patients and the potential for further benefit, additional investigation into both short-term and long-term patient outcomes is warranted.

\section{References}

1. Chiu J, Stechison MT. Percutaneous vertebral augmentation and reconstruction with an intravertebral mesh and morcelized bone graft. Surg Technol Int 2005;14:287-96.

2. Lam S, Khoo LT. A novel percutaneous system for bone graft delivery and containment for elevation and stabilization of vertebral compression fractures. Technical note. Neurosurg Focus 2005;18(3):e10.

3. Truumees E, Hilibrand A, Vaccaro AR. Percutaneous vertebral augmentation. Spine J 2004;4(2):218-29.

4. Taylor RS, Taylor RJ, Fritzell P. Balloon kyphoplasty and vertebroplasty for vertebral compression fractures: a comparative systematic review of efficacy and safety. Spine 2006;31(23):2747-55.

5. Watts NB, Harris ST, Genant HK. Treatment of painful osteoporotic vertebral Fractures with percutaneous vertebroplasty or kyphoplasty. Osteoporosis Int 2001;12:429-37.

6. Machinis TG, Fountas KN, Feltes $\mathrm{CH}$, et al. Pain outcome and vertebral body height restoration in patients undergoing kyphoplasty. Southern Med J 2006;99(5):457-60.

7. Kallmes DF, Jensen ME. Percutaneous vertebroplasty. Radiology 2003;229(1):27-36.

8. Garfin SR, Buckley RA, Ledlie J. Balloon kyphoplasty for symptomatic vertebral body compression fractures results in rapid, significant, and sustained improvements in back pain, function, and quality of life for elderly patients. Spine 2006;31(19):2213-20.

9. Fribourg D, Tang C, Sra P, et al. Incidence of subsequent vertebral fracture after kyphoplasty. Spine 2004;29(20):2270-6.

10. Pflugmacher R, Schroeder RJ, Klostermann CK. Incidence of adjacent vertebral fractures in patients treated with balloon kyphoplasty: two years' prospective follow-up. Acta Radiol 2006;47(8):830-40.

11. Khanna AJ, Reinhardt MK, Togawa D, et al. Functional outcomes of kyphoplasty for the treatment of osteoporotic and osteolytic vertebral compression fractures. Osteoporosis Int 2006;17(6):817-26.

12. Vogl TJ, Proschek D, Schwartz W, et al. CT-guided percutaneous vertebroplasty in the therapy of vertebral compression fractures. Eur Radiol 2006;16:797-803.

13. Vaccaro AR, Betz RB, Zeidman SM. Principles and practice of spine surgery. 2003. p. 374-375.

14. Wilke HJ, Mehnert U, Claes LE, et al. Biochemical evaluation of vertebroplasty and kyphoplasty with polymethyl methacrylate or calcium phosphate cement under cyclic loading. Spine 2006;31(25):2934-41.

15. Mitarai N, Nori F. Wet granular materials. Adv Phys 2006;55 (1-2):1-45.

16. Hollingworth W, Jarvik JG. Evidence on the effectiveness and cost-effectiveness of vertebroplasty: a review of policy makers' responses. Acad Radiol 2006;13(5):550-5. 